\title{
Student difficulties with the corrections to the energy spectrum of the hydrogen atom for the intermediate field Zeeman effect
}

\author{
Emily Marshman, Christof Keebaugh, and Chandralekha Singh \\ Department of Physics and Astronomy, University of Pittsburgh, Pittsburgh, PA 15260
}

\begin{abstract}
We discuss an investigation of student difficulties with the corrections to the energy spectrum of the hydrogen atom for the intermediate field Zeeman effect using the degenerate perturbation theory. The investigation was carried out in advanced quantum mechanics courses by administering free-response and multiple-choice questions and conducting individual interviews with students. We find that students share many common difficulties related to relevant physics concepts. In particular, students often struggled with mathematical sense-making in this context of quantum mechanics which requires interpretation of the implications of degeneracy in the unperturbed energy spectrum and how the Zeeman perturbation will impact the splitting of the energy levels. We discuss how the common difficulties often arise from the fact that applying linear algebra concepts correctly in this context with degeneracy in the energy spectrum is challenging for students.
\end{abstract}

\section{INTRODUCTION AND BACKGROUND}

Quantum mechanics (QM) is challenging even for upperlevel undergraduate and graduate students and students often struggle with the non-intuitive subject matter and in making connections between mathematics and QM concepts (e.g., see Refs. [1-10]). Prior research studies have found that students have difficulty connecting and applying mathematics correctly in introductory physics contexts (e.g., see Refs. [11-13]). Mathematical sense-making in the context of solving physics problems can often be more difficult than when solving equivalent mathematics problems without the physics context [1113]. Since working memory is constrained to a limited number of chunks and students' knowledge chunks pertaining to physics concepts are small when they are developing expertise, use of mathematics in physics can increase the cognitive load during problem solving especially if students are not proficient in the mathematics involved [14]. Therefore, students may struggle to integrate mathematics and physics concepts. Since mathematical sense-making while focusing on solving a physics problem is often more challenging, students sometimes make mathematical mistakes that they otherwise would not make if the physics context was absent [11-13].

One QM concept that involves connecting mathematics to a physical situation is degenerate perturbation theory (DPT) in the context of the Zeeman effect. We investigated student difficulties with finding the first-order corrections to the energies of the hydrogen atom for the Zeeman effect using DPT, which included probing of difficulties in mathematical sense making in this QM context so that the research can be used as a guide to develop learning tools to improve student understanding.

While the solution for the Time-Independent Schrödinger Equation (TISE) for the hydrogen atom with Coulomb potential energy can be obtained exactly, the TISE involving the Zeeman effect must also include the fine structure correction and cannot be solved exactly. However, since the finestructure and, in general, the Zeeman corrections to the energies are significantly smaller than the unperturbed energies, perturbation theory is an excellent method for computing the corrections to the energies. The high degree of symmetry of the unperturbed Hamiltonian leads to degeneracy in the energy spectrum and DPT must be used to find the perturbative corrections for the Zeeman effect.

The Hamiltonian $\hat{H}$ for the system can be expressed as the sum of two terms, the unperturbed Hamiltonian $\hat{H}^{0}$ and the perturbation $\hat{H}^{\prime}$, i.e., $\hat{H}=\hat{H}^{0}+\hat{H}^{\prime}$. The TISE for the unperturbed Hamiltonian, $\hat{H}^{0} \psi_{n}^{0}=E_{n}^{0} \psi_{n}^{0}$, is exactly solvable, where $\psi_{n}^{0}$ is the $n^{\text {th }}$ unperturbed energy eigenstate and $E_{n}^{0}$ is the unperturbed energy. The $n^{t h}$ energy can be approximated as $E_{n}=E_{n}^{0}+E_{n}^{1}+E_{n}^{2}+\ldots$ where $E_{n}^{i}$ for $i=1,2,3$. are the $i^{\text {th }}$ order corrections to the $n^{\text {th }}$ energy of the system. In perturbation theory, the first-order corrections to the energies are $E_{n}^{1}=\left\langle\psi_{n}^{0}\left|\hat{H}^{\prime}\right| \psi_{n}^{0}\right\rangle$ and the firstorder corrections to the unperturbed energy eigenstates are $\left|\psi_{n}^{1}\right\rangle=\sum_{m \neq n} \frac{\left\langle\psi_{m}^{0}\left|\hat{H}^{\prime}\right| \psi_{n}^{0}\right\rangle}{\left(E_{n}^{0}-E_{m}^{0}\right)}\left|\psi_{m}^{0}\right\rangle$, in which $\left\{\left|\psi_{n}^{0}\right\rangle\right\}$ is a complete set of eigenstates of the unperturbed Hamiltonian $\hat{H}^{0}$. If the eigenvalue spectrum of $\hat{H}^{0}$ has degeneracy, the perturbative corrections are only valid provided one uses a good basis [15]. For a given $\hat{H}^{0}$ and $\hat{H}^{\prime}$, a good basis consists of a complete set of eigenstates of $\hat{H}^{0}$ that diagonalizes $\hat{H}^{\prime}$ in each degenerate subspace of $\hat{H}^{0}$.

Using standard notations, the unperturbed Hamiltonian $\hat{H}^{0}$ of a hydrogen atom is $\hat{H}^{0}=\frac{\hat{p}^{2}}{2 m}-\frac{e^{2}}{4 \pi \epsilon_{0}} \frac{1}{r}$, which accounts only for the interaction of the electron with the nucleus via Coulomb attraction. The solution for the TISE for the hydrogen atom with Coulomb potential energy gives the unperturbed energies $E_{n}^{0}=-\frac{13.6 \mathrm{eV}}{n^{2}}$, where $n$ is the principal quantum number. The perturbation is $\hat{H}^{\prime}=\hat{H}_{f s}^{\prime}+\hat{H}_{Z}^{\prime}$, in which $\hat{H}_{Z}^{\prime}$ is the Zeeman term and $\hat{H}_{f s}^{\prime}$ is the fine structure term. The Zeeman term is given by $\hat{H}_{Z}^{\prime}=\frac{\mu_{B} B_{e x t}}{\hbar}\left(\hat{L}_{z}+2 \hat{S}_{z}\right)$ in which $\vec{B}_{\text {ext }}=B_{\text {ext }} \hat{z}$ is a uniform, time independent external magnetic field along the $\hat{z}$-direction, $\mu_{B}$ is the Bohr magneton and $\hat{L}_{z}$ and $\hat{S}_{z}$ are the operators corresponding to the $\mathrm{z}$ component of the orbital and spin angular momenta, respectively. The fine structure term includes the spin-orbit coupling and a relativistic correction for the kinetic energy, and is expressed as $\hat{H}_{f s}^{\prime}=\hat{H}_{r}^{\prime}+\hat{H}_{S O}^{\prime}$. Here, $\hat{H}_{r}^{\prime}=-\frac{\hat{p}^{4}}{8 m^{3} c^{2}}$ is the relativistic correction term and $\hat{H}_{S O}^{\prime}=\frac{e^{2}}{8 \pi \epsilon_{0}} \frac{1}{m^{2} c^{2} r^{3}} \vec{S} \cdot \vec{L}$ is the spin-orbit interaction term (all notations are standard).

We note that the unperturbed Hamiltonian is spherically 
symmetric since $\left[\hat{H}^{0}, \hat{\vec{L}}\right]=0$. Therefore, for a fixed $n, \hat{H}^{0}$ for the hydrogen atom is diagonal when any complete set of orthogonal states is chosen for the angular part of the basis (consisting of the product states of orbital and spin angular momenta). Thus, so long as the radial part of the basis is always chosen to be a stationary state wavefunction $R_{n l}(r)$ for the unperturbed hydrogen atom (for given principal and azimuthal quantum numbers $n$ and $l$ ), which we will assume throughout, the choice of a good basis amounts to choosing the angular part of the basis (angular basis) appropriately, i.e., ensuring that the perturbation is diagonal in each degenerate subspace of $\hat{H}^{0}$. Therefore, we focus on the angular basis to find a good basis and the corrections to the energies for the Zeeman effect. For the angular basis for each $n$, states in the coupled representation $\left|l, j, m_{j}\right\rangle$ are labeled by the quantum numbers $l, j$, and $m_{j}$ (they are eigenstates of $\hat{J}^{2}$ and $\hat{J}_{z}$ ) and the total angular momentum is defined as $\vec{J}=\vec{L}+\vec{S}$ (all notations are standard and $s$ has been suppressed from the states $\left|l, j, m_{j}\right\rangle$ since $s=1 / 2$ is a fixed value for a hydrogen atom). On the other hand, states $\left|l, m_{l}, m_{s}\right\rangle$ in the uncoupled representation are labeled by the quantum numbers $l, m_{l}$, and $m_{s}$ (notations are standard) and are eigenstates of $\hat{L}_{z}$ and $\hat{S}_{z}$.

An angular basis consisting of states in the coupled representation forms a good basis for the fine structure term $\hat{H}_{f s}^{\prime}$ since with this choice of the angular basis, $\hat{H}_{f s}^{\prime}$ is diagonal in each degenerate subspace of $\hat{H}^{0}$. But a basis consisting of states in the uncoupled representation forms a good basis for the Zeeman perturbation $\hat{H}_{Z}^{\prime}$. (In this case with $\hat{H}_{Z}^{\prime}$ only, first order PT yields the exact result since $\left[\hat{H}^{0}, \hat{H}_{Z}^{\prime}\right]=0$.) Therefore, for the intermediate field Zeeman effect, in which $\hat{H}^{\prime}=\hat{H}_{f s}^{\prime}+\hat{H}_{Z}^{\prime}$ and $\hat{H}_{f s}^{\prime}$ and $\hat{H}_{Z}^{\prime}$ are treated on equal footing (i.e., energy corrections due to the two terms are comparable $E_{f s}^{\prime} \approx E_{Z}^{\prime}$ ), neither a basis consisting of states in the coupled representation nor a basis consisting of states in the uncoupled representation forms a good angular basis to find perturbative corrections for the hydrogen atom placed in an external magnetic field. For example, in a basis consisting of states in the coupled representation $\left(\left|l, j, m_{j}\right\rangle\right.$ ), the perturbation matrix $\hat{H}^{\prime}=\hat{H}_{Z}^{\prime}+\hat{H}_{f s}^{\prime}$ corresponding to the $n=2$ subspace is given below (in which $\gamma=\left(\frac{\alpha}{8}\right)^{2} 13.6$ $\mathrm{eV}, \alpha=\frac{e^{2}}{4 \pi \epsilon_{0} \hbar c}, \beta=\mu_{B} B_{\text {ext }}$ and the basis states are chosen in the order $\left|0, \frac{1}{2}, \frac{1}{2}\right\rangle,\left|0, \frac{1}{2},-\frac{1}{2}\right\rangle,\left|1, \frac{3}{2}, \frac{3}{2}\right\rangle,\left|1, \frac{3}{2},-\frac{3}{2}\right\rangle$, $\left|1, \frac{3}{2}, \frac{1}{2}\right\rangle,\left|1, \frac{1}{2}, \frac{1}{2}\right\rangle,\left|1, \frac{3}{2},-\frac{1}{2}\right\rangle$, and $\left.\left|1, \frac{1}{2},-\frac{1}{2}\right\rangle\right)$ : $\hat{H}^{\prime}=\left[\begin{array}{cccccccc}5 \gamma-\beta & 0 & 0 & 0 & 0 & 0 & 0 & 0 \\ 0 & 5 \gamma+\beta & 0 & 0 & 0 & 0 & 0 & 0 \\ 0 & 0 & \gamma-2 \beta & 0 & 0 & 0 & 0 & 0 \\ 0 & 0 & 0 & \gamma+2 \beta & 0 & 0 & 0 & 0 \\ 0 & 0 & 0 & 0 & \gamma-\frac{2}{3} \beta & \frac{\sqrt{2}}{3} \beta & 0 & 0 \\ 0 & 0 & 0 & 0 & \frac{\sqrt{2}}{3} \beta & 5 \gamma-\frac{1}{3} \beta & 0 & 0 \\ 0 & 0 & 0 & 0 & 0 & 0 & \gamma+\frac{2}{3} \beta & \frac{\sqrt{2}}{3} \beta \\ 0 & 0 & 0 & 0 & 0 & 0 & \frac{\sqrt{2}}{3} \beta & 5 \gamma+\frac{1}{3} \beta\end{array}\right]$.

The following procedure describes what students should be able to do to determine a good basis and find the first order corrections to the energy spectrum for the Zeeman effect: (1) choose an initial basis consisting of a complete set of eigenstates of $\hat{H}^{0}$, (2) write the $\hat{H}^{0}$ and $\hat{H}^{\prime}$ matrices in the initially chosen basis, (3) recognize $\hat{H}^{\prime}$ in each degenerate subspace of $\hat{H}^{0}$, (4) diagonalize the $\hat{H}^{\prime}$ matrix in each degenerate subspace of $\hat{H}^{0}$ to determine a good basis, and (5) identify and be able to explain why the first-order corrections to the energy spectrum are the diagonal matrix elements of the $\hat{H}^{\prime}$ matrix given by $E_{n}^{1}=\left\langle\psi_{n}^{0}\left|\hat{H}^{\prime}\right| \psi_{n}^{0}\right\rangle$ in the good basis.

\section{METHODOLOGY}

Student difficulties with the corrections to the energies of the hydrogen atom for the Zeeman effect using DPT were investigated using five years of data involving responses from 64 upper-level undergraduate students and 42 first-year graduate students to open-ended and multiple-choice questions administered in-class after traditional instruction in relevant concepts. The undergraduates were in an upper-level QM course, and graduate students were in a graduate-level QM course. Additional insight about the difficulties was gained from 13 individual think-aloud interviews (a total of 45 hours) with undergraduate and graduate students following the completion of their quantum mechanics courses. Students were provided with all relevant information discussed in the introduction section and had lecture-based instruction in relevant concepts. Similar percentages of undergraduate and graduate students displayed difficulties with DPT.

We first analyzed responses of 32 undergraduates on questions related to DPT in the context of the Zeeman effect for the hydrogen atom administered in two previous years. Then, we examined the difficulties that 32 undergraduate and 42 graduate students had with identifying a good basis for the Zeeman effect in the following three years as part of an in-class quiz after traditional lecture-based instruction. The following question is representative of a series of questions that were posed after traditional lecture-based instruction on relevant concepts (the operator $\hat{H}^{\prime}$, in Q1, is a proxy for the operators $\hat{H}_{f s}^{\prime}, \hat{H}_{Z}^{\prime}$, and $\hat{H}_{f s}^{\prime}+\hat{H}_{Z}^{\prime}$ that were listed individually in three separate questions):

Q1. A perturbation $\hat{H}^{\prime}$ acts on a hydrogen atom with the unperturbed Hamiltonian $\hat{H}^{0}=-\frac{\hbar^{2}}{2 m} \nabla^{2}-\frac{e^{2}}{4 \pi \epsilon_{0}}\left(\frac{1}{r}\right)$. For the perturbation Hamiltonian $\hat{H}^{\prime}$, circle $\mathbf{A L L}$ of the representations that form the angular part of a good basis and explain your reasoning. Assume that for all cases the principal quantum number is fixed to $n=2$.

i. Coupled representation,

ii. Uncoupled representation,

iii. Any arbitrary complete orthonormal basis constructed with linear combinations of states in the coupled representation with the same l (i.e., states with different l values are not mixed),

iv. Any arbitrary complete orthonormal basis constructed with linear combinations of states in the uncoupled representation with the same $l$ (i.e., states with different $l$ values are not mixed),

v. Neither coupled nor uncoupled representation.

In order to find the perturbative corrections, one must first choose a good basis. Q1 focuses on the bases that form a good angular basis for the perturbation $\hat{H}^{\prime}=\hat{H}_{f s}^{\prime}+\hat{H}_{Z}^{\prime}$, as well 
as the perturbations $\hat{H}_{f s}^{\prime}$ and $\hat{H}_{Z}^{\prime}$ individually. Knowledge of the bases that form a good angular basis for the individual perturbations $\hat{H}_{f s}^{\prime}$ and $\hat{H}_{Z}^{\prime}$ can be helpful when determining a good basis for $\hat{H}^{\prime}=\hat{H}_{f s}^{\prime}+\hat{H}_{Z}^{\prime}$.

The unperturbed Hamiltonian $\hat{H}^{0}$ is spherically symmetric with unperturbed energies only dependent on $n$ and therefore options i, ii, iii, and iv in Q1 all form a complete set of angular eigenstates of $\hat{H}^{0}$. Therefore, one must consider which set of basis states in Q1 also diagonalize the given perturbation $\hat{H}^{\prime}$ in each degenerate subspace of the unperturbed Hamiltonian $\hat{H}^{0}$. In each degenerate subspace of $\hat{H}^{0}$, the fine structure term $\hat{H}_{f s}^{\prime}$ is diagonal if the basis is chosen to consist of states in the coupled representation (option i in Q1) and the Zeeman term is diagonal if the basis is chosen to consist of states in the uncoupled representation (option ii in Q1), but not vice versa. Therefore, for the intermediate field Zeeman effect with $\hat{H}^{\prime}=\hat{H}_{f s}^{\prime}+\hat{H}_{Z}^{\prime}$, neither a basis consisting of states in the coupled representation nor a basis consisting of states in the uncoupled representation forms a good basis and option $\mathrm{v}$ in Q1 is correct. In order to determine a good basis, one may first choose a basis, e.g., consisting of states in either the coupled or uncoupled representation and then diagonalize the perturbation $\hat{H}^{\prime}=\hat{H}_{f s}^{\prime}+\hat{H}_{Z}^{\prime}$ in the $n=2$ degenerate subspace of the unperturbed Hamiltonian $\hat{H}^{0}$.

\section{STUDENT DIFFICULTIES}

Students had some difficulties with DPT in general (not restricted to the context of the Zeeman effect only). For example, when students were asked to determine a good basis for finding the corrections to the energies of the hydrogen atom, some students did not even realize that DPT should be used. Other students knew that they had to use DPT to find the corrections to the wavefunction, but they did not use DPT to find the first-order corrections to the energies. These students often incorrectly claimed that they did not need to use DPT since no terms in $E_{n}^{1}=\left\langle\psi_{n}^{0}\left|\hat{H}^{\prime}\right| \psi_{n}^{0}\right\rangle$ "blow up". Other students only focused on the Zeeman term $\hat{H}_{Z}^{\prime}$ when asked to determine a good basis for finding the corrections to the energies. In particular, they did not take into account the fine structure term $\hat{H}_{f s}^{\prime}$. However, the fine structure term must be considered when determining the corrections to the unperturbed energy spectrum for the Zeeman effect.

In response to Q1, students struggled to realize that neither a basis consisting of states in the coupled representation nor a basis consisting of states in the uncoupled representation forms a good basis for the perturbative corrections to the hydrogen atom placed in an external magnetic field. The results are summarized in Table I. Table I shows that only $44 \%$ of undergraduates and $33 \%$ of graduate students correctly identified that option $\mathrm{v}$ in Q1 is the correct answer for the Zeeman effect. Additionally, $16 \%$ of undergraduate and $17 \%$ of graduate students did not provide any answer to question Q1 after traditional instruction in relevant concepts.

Below, we discuss student difficulties in selecting the representation that forms a good basis in Q1 and finding the correc-
TABLE I. The percentages of undergraduate $(\mathrm{U})$ and graduate $(\mathrm{G})$ students who chose the options i-v in Q1 for the perturbation $\hat{H}^{\prime}=$ $\hat{H}_{f s}^{\prime}+\hat{H}_{Z}^{\prime}$ after traditional instruction for undergraduates (U) (number of students $N=32)$ and graduate students $(\mathrm{G})(N=42)$.

\begin{tabular}{|c|c|c|c|c|c|c|}
\hline & i & ii & iii & iv & v & Blank \\
\hline $\mathrm{U}(\%)$ & 28 & 22 & 16 & 13 & 44 & 16 \\
\hline $\mathrm{G} \mathrm{( \% )}$ & 29 & 17 & 12 & 12 & 33 & 17 \\
\hline
\end{tabular}

tions to the energy spectrum, based primarily upon responses during the think aloud interviews.

A. Difficulty understanding why diagonalizing the entire $\hat{H}^{\prime}$ matrix is problematic: Many students did not realize that when the initially chosen basis is not a good basis and the unperturbed Hamiltonian $\hat{H}^{0}$ and the perturbing Hamiltonian $\hat{H}^{\prime}=\hat{H}_{f s}^{\prime}+\hat{H}_{Z}^{\prime}$ do not commute, they must diagonalize the $\hat{H}^{\prime}=\hat{H}_{f s}^{\prime}+\hat{H}_{Z}^{\prime}$ matrix only in each degenerate subspace of $\hat{H}^{0}$. When presented with a similar system and asked to determine the perturbative corrections, one interviewed student who attempted to diagonalize the entire $\hat{H}^{\prime}$ matrix justified his reasoning by incorrectly stating, "We must find the simultaneous eigenstates of $\hat{H}^{0}$ and $\hat{H}^{\prime}$." Discussions suggest that this student, and others with similar difficulties often did not realize that when $\hat{H}^{0}$ and $\hat{H}^{\prime}=\hat{H}_{f s}^{\prime}+\hat{H}_{Z}^{\prime}$ do not commute, we cannot simultaneously diagonalize $\hat{H}^{0}$ and $\hat{H}^{\prime}=\hat{H}_{f s}^{\prime}+\hat{H}_{Z}^{\prime}$ since they do not share a complete set of eigenstates. Students struggled with the fact that if $\hat{H}^{0}$ and $\hat{H}^{\prime}=\hat{H}_{f s}^{\prime}+\hat{H}_{Z}^{\prime}$ do not commute, diagonalizing $\hat{H}^{\prime}=\hat{H}_{f s}^{\prime}+\hat{H}_{Z}^{\prime}$ produces a basis in which $\hat{H}^{0}$ is not diagonal. However, since $\hat{H}^{0}$ is the dominant term and $\hat{H}^{\prime}=\hat{H}_{f s}^{\prime}+\hat{H}_{Z}^{\prime}$ provides only small corrections, we must ensure that the basis states used to determine the perturbative corrections remain eigenstates of $\hat{H}^{0}$.

B. Incorrectly claiming that BOTH a basis consisting of states in the coupled representation and a basis consisting of states in the uncoupled representation are good bases: In Q1, many students correctly identified that the good basis for the fine structure term $\hat{H}_{f s}^{\prime}$ is a basis consisting of states in the coupled representation (option i) and also correctly identified that the good basis for the Zeeman term $\hat{H}_{Z}^{\prime}$ is a basis consisting of states in the uncoupled representation (option ii in Q1). However, after correctly identifying the good basis for the two perturbations individually, some students did not realize that neither the coupled nor the uncoupled representation (option v in Q1) forms a good basis for the Zeeman effect in which the perturbation is $\hat{H}^{\prime}=\hat{H}_{f s}^{\prime}+\hat{H}_{Z}^{\prime}$. One interviewed student incorrectly claimed that "the coupled are a good basis for $\hat{H}_{f s}^{\prime}$ and uncoupled are a $\operatorname{good}$ basis for $\hat{H}_{Z}^{\prime}$, so both coupled and uncoupled form a $\operatorname{good}$ basis for $\hat{H}_{f s}^{\prime}+\hat{H}_{Z}^{\prime}$." This student and others with this type of response thought that since a basis consisting of states in the coupled representation (option i in Q1) forms a good basis for the fine structure term $\hat{H}_{f s}^{\prime}$ and a basis consisting of states in the uncoupled representation (option ii in Q1) forms a good basis for the Zeeman term $\hat{H}_{Z}^{\prime}$, a good basis for the perturbation consisting of the sum of these two perturbations is either a basis consisting of 
states in the coupled or uncoupled representation.

C. Incorrectly claiming that a $\operatorname{good}$ basis does not exist for the Zeeman effect: Some students argued that good basis does not exist for the intermediate field Zeeman effect and struggled to realize that the coupled representation or the uncoupled representation are not the only two possibilities for the angular basis. One interviewed student with this type of reasoning had difficulty understanding options iii and iv in Q1, stating: "I don't know what a linear combination of coupled or uncoupled states is. I thought there were just coupled states or uncoupled states." This student and others with this type of reasoning did not realize that a good basis could be constructed from a linear combination of states in the coupled or uncoupled representation.

Some students had difficulty realizing that any linear combination of states from the same degenerate subspace of $\hat{H}^{0}$ are eigenstates of $\hat{H}^{0}$. For example, one student who correctly identified that neither the coupled nor the uncoupled representation forms a good basis for the Zeeman effect argued that "no good basis exists since we cannot diagonalize a part of the $\hat{H}^{\prime}$ matrix $\left(\hat{H}^{\prime}\right.$ in the degenerate subspace of $\left.\hat{H}^{0}\right)$ without affecting the $\hat{H}^{0}$ matrix." This student and others who provided similar incorrect reasoning claimed that by diagonalizing $\hat{H}^{\prime}$ in the degenerate subspace of $\hat{H}^{0}$, the $\hat{H}^{0}$ matrix would no longer be diagonal. However, due to the degeneracy, any linear combination of states from the same degenerate subspace of $\hat{H}^{0}$ are eigenstates of $\hat{H}^{0}$. Therefore, diagonalizing $\hat{H}^{\prime}$ in the degenerate subspace of $\hat{H}^{0}$ determines the special linear combination that forms a good basis.

D. Incorrectly claiming that the choice of the initial basis affects corrections to the energy spectrum: Of the students who correctly identified that a good basis for the Zeeman effect consists of special linear combinations of states in the coupled or uncoupled representation, some did not realize that the first order corrections to the energy spectrum would be the same regardless of the initial choice of the basis. A good basis cannot easily be identified at the onset. In order to determine a good basis and the first order corrections to the energy spectrum due to the Zeeman effect, one can initially choose a basis consisting of states in the coupled representation and then diagonalize $\hat{H}^{\prime}=\hat{H}_{f s}^{\prime}+\hat{H}_{Z}^{\prime}$ in each degenerate subspace of $\hat{H}^{0}$. However, one could also initially choose a basis consisting of states in the uncoupled representation and then diagonalize $\hat{H}^{\prime}=\hat{H}_{f s}^{\prime}+\hat{H}_{Z}^{\prime}$ in each degenerate subspace of $\hat{H}^{0}$ to determine a good basis and the first order corrections to the energy spectrum due to the Zeeman effect. Regardless of the choice of the initial basis, after diagonalizing $\hat{H}^{\prime}=\hat{H}_{f s}^{\prime}+\hat{H}_{Z}^{\prime}$ in each degenerate subspace of $\hat{H}^{0}$, the first order corrections to the energy spectrum due to the Zeeman effect will be the same in any good basis. Many students thought that the first order corrections to the energies depend on the initial choice of basis. Therefore, if one chooses a basis consisting of states in the coupled representation, then the first order corrections in this case would be different than those obtained had a basis consisting of states in the uncoupled representation been chosen as the initial basis. However, it does not make sense experimentally that the observed perturbative corrections would depend upon the choice of basis. Lack of appropriate connection between physics and mathematics in the context of DPT for the Zeeman effect sheds light on student epistemology and the difficulty in mathematical sense-making in QM [11].

\section{SUMMARY AND FUTURE PLAN}

Both upper-level undergraduate and graduate students struggled with finding perturbative corrections to the hydrogen atom energy spectrum for the intermediate field Zeeman effect using DPT. Interviewed students' responses suggested that some of them held epistemological beliefs inconsistent with the framework of QM and struggled with mathematical sense-making in the context of QM in which the paradigm is novel [4]. After traditional instruction, some students claimed that different initial choices of the basis before a good basis has been found will yield different corrections to the energy spectrum of the hydrogen atom for the Zeeman effect. These students had difficulty in connecting experimental observations with quantum theory and in correctly reasoning that since the corrections to the energy spectrum can be measured experimentally, different choices of the initial basis cannot yield different physically observable corrections to the energy spectrum. Since students are still developing expertise in QM and the DPT requires appropriate integration of mathematical and physical concepts, cognitive overload can be high while reasoning about these problems [14]. Many advanced students found it challenging to do metacognition [14] in this context of QM and provided responses that were not consistent with each other. We are using the difficulties as a guide in developing a Quantum Interactive Learning Tutorial (QuILT) to help students develop a good grasp of these concepts.

\section{ACKNOWLEDGMENTS}

We thank the NSF for awards PHY-1505460 and 1806691.
[1] C. Singh, Am. J. Phys. 69, 885 (2001).

[2] D. Zollman, S. Rebello, K. Hogg, Am. J. Phys. 70, 252 (2002).

[3] M. Wittmann, R. Steinberg, and E. Redish, Am. J. Phys. 70, 218 (2002).

[4] E. Marshman, C. Singh, Phys. Rev. ST PER 11, 020119 (2015).

[5] C. Singh, M. Belloni, W. Christian, Phys.Today 59, 43 (2006).

[6] Keebaugh, Marshman, Singh, Euro. J. Phys. 39, 045701 (2018).

[7] C. Singh, Am. J. Phys. 76, 277 (2008).
[8] E. Marshman, and C. Singh, Euro. J. Phys. 39, 015707 (2018).

[9] Keebaugh, Marshman, Singh, Euro. J. Phys. 39, 055701 (2018).

[10] C. Singh, E. Marshman, Phys. Rev. ST PER 11, 020117 (2015).

[11] J. Tuminaro, E. Redish, Phys. Rev. ST PER 3, 020101 (2007).

[12] A. Maries, S. Lin, C. Singh, Phys. Rev. PER 13, 020103 (2017).

[13] J. Li and C. Singh, AIP Proc. 1413, 243 (2012).

[14] J. Sweller, Learning and Instruction 4, 295 (1994).

[15] D. Griffiths, Introduction to Quantum Mechanics (2005). 